\title{
Safety and feasibility of simultaneous ipsilateral proximal carotid artery stenting and cerebral aneurysm coiling
}

\author{
Aamir Badruddin',2, Mohamed S. Teleb' , Michael G. Abraham1', Muhammad A. Taqi' and Osama O. Zaidat ${ }^{1,3,4 *}$ \\ 1 Department of Neurology, Froedtert \& The Medical College of Wisconsin, Milwaukee, WI, USA \\ 2 Daniel M. Soref Clinical Neuroscience Fellow at Froedtert Hospital, Froedtert \& The Medical College of Wisconsin, Milwaukee, WI, USA \\ ${ }^{3}$ Department of Neurosurgery, Froedtert \& The Medical College of Wisconsin, Milwaukee, WI, USA \\ ${ }^{4}$ Department of Radiology, Froedtert \& The Medical College of Wisconsin, Milwaukee, WI, USA
}

\section{Edited by:}

Syed I. Hussain, Michigan State

University, USA

\section{Reviewed by:}

Syed I. Hussain, Michigan State

University, USA

Shazam Hussain, Cleveland Clinic

Foundation, USA

\section{${ }^{*}$ Correspondence:}

Osama O. Zaidat, Neurointerventional Program, Medical College of Wisconsin and Froedtert Hospital West, $9200 \mathrm{~W}$.

Wisconsin Ave, Milwaukee, WI 53226,

USA.

e-mail: szaidat@mcw.edu
Coexistence of cerebral aneurysm and carotid artery disease may be encountered in clinical practice. Theoretical increase in aneurysmal blood flow may increase risk of rupture if carotid artery disease is treated first. If aneurysm coiling is performed first, stroke risk may increase while repeatedly crossing the diseased artery. It is controversial which disease to treat first, and whether it is safe to treat both simultaneously via endovascular procedures. We document the safety and feasibility of such an approach. Review of collected neurointerventional database at our institution was performed for patients who underwent both carotid artery stenting (CAS) and aneurysm coil embolization (ACE) simultaneously. All patients underwent carotid stenting followed by aneurysm coiling in the same setting. Demographic, clinical data, and outcome measures including success rate and periprocedural complications were collected. Five hundred and ninety aneurysms coiling were screened for patients who underwent combined CAS and ACE. Ten patients were identified. Mean age was 67.7 years (range 51-89). The success rate for stenting and coiling was $100 \%$ with no immediate complications. The coiling procedure time was extended by an average of $45 \mathrm{~min}$ for performing both procedures jointly. No stroke, TIAs, or aneurysmal rebleeding was found on their most recent follow up. Our case series demonstrates that it is safe and feasible to perform CAS and ACE simultaneously as one procedure which may avoid unwanted risk of treating either disease at two separate time sessions.

Keywords: carotid stenting, aneurysm, coil embolization

\section{INTRODUCTION}

The simultaneous occurrence of intracranial aneurysm and carotid artery stenosis is around 3\% (Pappada et al., 1997; Kappelle et al., 2000). Certain risk factors are shared between carotid atherosclerosis and cerebral aneurysms. These include age, hypertension, and tobacco use (NASCET Trial Investigators, 1991; Wiebers et al., 2003).

Theoretical increase in aneurysmal blood flow may increase risk of rupture if carotid artery disease is treated first. If aneurysm coiling is performed first, stroke risk may increase while repeatedly crossing the diseased artery. This brings an important management and clinical decision approach. Are we putting patients at risk by treating each disease in isolation? When aneurysm and proximal large vessel stenosis occur in the same arterial tree, it may be feasible to treat both lesions via endovascular technique without added morbidity.

There are few case series documenting open surgical approach to the treatment of concomitant lesions (Pappada et al., 1996; Porter et al., 1997). And, our review found only one case report documenting endovascular treatment of both lesions in one session (Navaneethan et al., 2006). This article aims to discuss safety and feasibility of endovascular treatment of unruptured cerebral aneurysms and ipsilateral carotid artery stenosis. Some surgical considerations are also reviewed.

\section{MATERIALS AND METHODS}

We reviewed a neurointerventional database at our institution for patients who underwent carotid artery stenting (CAS) and aneurysm coil embolization (ACE) in one operative session. Carotid artery stenosis was measured using the NASCET criteria. Aneurysm dimensions were measured using automatically calibrated software available with the angiographic equipment. During the procedure, the blood pressure was strictly monitored and treated and all patients were anticoagulated intra-procedurally using unfractionated heparin was administered to achieve activated clotting time (ACT) of 250-300 s. All patients were treated with aspirin and clopidogrel perioperatively and the antiplatelet treatment was continued for a minimum of 3 months.

Patient with subarachnoid hemorrhage (SAH) were treated with antiplatelet agents after securing the aneurysm. In these cases as soon as the first coil was placed, unfractionated heparin was administered intravenously to achieve ACT of 250-300 s.

In all cases the carotid artery was treated first. A 6 French sheath was placed in the common carotid artery while the carotid artery lesion was crossed with a microwire and a microcatheter. A distal emboli protection device was placed in the distal cervical segment of the internal carotid artery. Carotid artery stent was placed across the lesion and post stenting angioplasty was performed if needed. After stenting the distal emboli protection was removed and the 
guide catheter was advanced distally into the internal carotid artery traversing the stent once. A microcatheter was introduced into the aneurysm with the aid of a microwire and the coiling was performed with angiographically satisfactory outcome. The microcatheter and guide catheter were subsequently removed from the body. (see Figure 1).

\section{RESULTS}

Ten cases were treated between July 2005 and May 2010 (Table 1). Patients underwent CAS ipsilateral to the site of aneurysm or in the case of anterior communicating artery, internal carotid artery with the dominant anterior cerebral artery prior to aneurysm coiling. The mean age was $67.7 \pm 12.2$ years (range $51-89$ ), all were Caucasian, and one was male. Carotid artery stenosis was always present at the level of the bulb and was at least $75 \%$ in all cases. Mean stenosis was $79 \pm 6.3 \%$ (range 75-95). In seven patients the aneurysms were discovered after undergoing imaging for complaints of headache or TIA. Two patients presented with SAH and one patient had symptomatic re-stenosis of ICA after a previous carotid endarterectomy. There were no procedure related complications during either of the treatments or in the perioperative period. All but one patient had imaging follow-up. Mean time to follow-up was $7.5 \pm 5.5$ months (range 3-22 months). Follow up imaging of these patients did not reveal any re-stenosis, aneurysm expansion, or coil

Table 1 | Demographic and angiographic data.

\begin{tabular}{|c|c|c|c|c|c|c|c|}
\hline Age & Sex & $\begin{array}{l}\text { Stenosis } \\
\text { location }\end{array}$ & $\begin{array}{l}\text { Stenosis } \\
(\%)\end{array}$ & $\begin{array}{l}\text { Aneurysm } \\
\text { size (mm) }\end{array}$ & $\begin{array}{l}\text { Aneurysm } \\
\text { location }\end{array}$ & Complications & $\begin{array}{l}\text { Presenting } \\
\text { symptom }\end{array}$ \\
\hline 56 & $\mathrm{~F}$ & LICA & 95 & $5 \times 4$ & LMCA & None & TIA \\
\hline 62 & $\mathrm{~F}$ & RICA & 75 & $6.5 \times 4.5$ & A Comm & None & Headache \\
\hline 79 & $\mathrm{~F}$ & LICA & 75 & $7 \times 5$ & Supraclinoid ICA & None & Headache \\
\hline 67 & $\mathrm{~F}$ & R ICA & 81 & $8 \times 5$ & Supraclinoid ICA & None & Incidental \\
\hline 72 & $\mathrm{~F}$ & LICA & 76 & $8 \times 6$ & A Comm & None & Headache \\
\hline 78 & $\mathrm{~F}$ & LICA & 83 & $5 \times 6$ & LMCA & None & SAH \\
\hline 69 & $\mathrm{~F}$ & LICA & 75 & $4.5 \times 5.5$ & LMCA & None & Headache \\
\hline
\end{tabular}
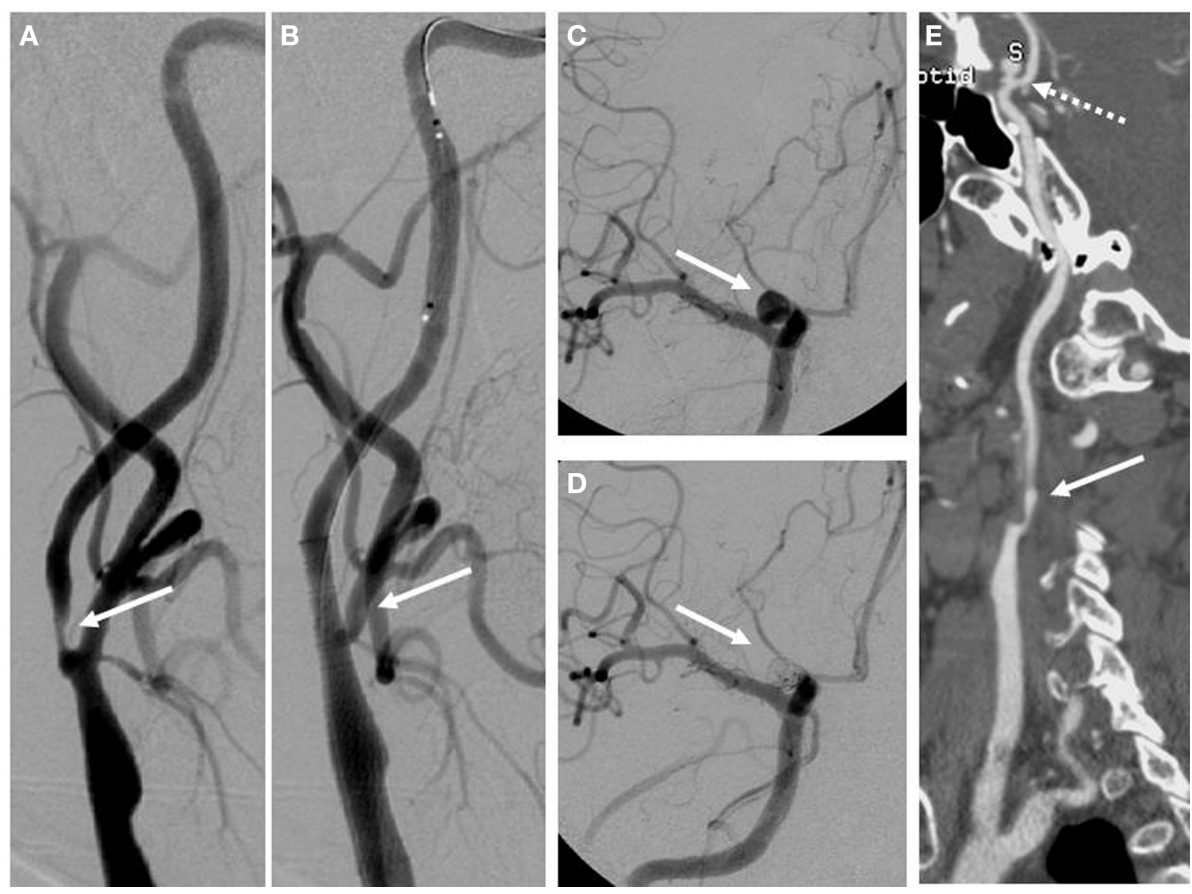

FIGURE 1 | Digital subtraction angiography of pre and post treatment internal carotid arteries is seen in $(A, B)$ respectively. Pre and post treatment aneurysms are seen in the same arterial tree in (C,D). A digitally reconstructed CT angiogram demonstrated the carotid atherosclerotic disease (solid arrow) and the unsecured aneurysm (dashed arrow) distal to it in (E). 
compaction. Eight patients had digital subtraction angiography performed within 12 months of treatment. One patient had MRA only follow up at 5 months.

One patient who had presented with SAH died 1 week after treatment due to complications of SAH and family's decision to withdraw care.

\section{DISCUSSION}

When clinician and interventionalist encounter ipsilateral carotid disease and cerebral aneurysm, they face a very critical patient safety issue of which one to treat first. Treatment decision for a carotid stenosis and an intracranial cerebral aneurysm simultaneously is complex as the treatment poses several risks. It is important to note that in this series all treated carotid lesions were ipsilateral. This fact has surgical implications as traversing a stenosed and diseased segment repeatedly would add to the complication risk. Therefore, in most cases the rationale for treating the lesion was to afford improved access to the aneurysm and reduce the procedural risk. In this group the aneurysm was found incidentally due to headache or a TIA located in the territory of the untreated vessel; and aneurysm was the primary discovery and the carotid disease was a secondary one.

The other group of patients was in whom the stenosis was discovered first due to a TIA or an ischemic stroke, and the aneurysm was found incidentally. If the aneurysm was discovered first then the ipsilateral carotid had to be treated (even if it is asymptomatic) for endovascular surgical access to the aneurysm. If the patient underwent carotid stenting for a symptomatic carotid lesion then the distal aneurysm in its territory could not be left unprotected due to increased flow and increased theoretical risk of rupture. Therefore, regardless of the lesion of primary concern and manner of presentation, concomitant treatment of the lesions would be prudent.

From a safety point of view, the risk of hematoma, stroke, TIA, or infection from a second procedure is consolidated by performing a single procedure rather than two.

Certain issues complicate simultaneous treatment of these lesions. First, treatment of an unruptured aneurysm is dictated by its risk of rupture, which in turn is dictated by the aneurysm's size

\section{REFERENCES}

ACAS Trial investigators. (1995). Endarterectomy for asymptomatic carotid artery stenosis. Executive Committee for the Asymptomatic Carotid Atherosclerosis Study. JAMA 273, 1421-1428.

Kappelle, L. J., Eliasziw, M., Fox, A. J., and Barnett, H. J. (2000). Small, unruptured intracranial aneurysms and management of symptomatic carotid artery stenosis. North American Symptomatic Carotid Endarterectomy Trial Group. Neurology 55, 307-309.

NASCETTrial Investigators. (1991).Clinical alert: benefit of carotid endarterectomy for patients with high-grade stenosis of the internal carotid artery. National Institute of Neurological Disorders and Stroke and Trauma Division. North American Symptomatic Carotid

and location as shown by the ISUIA study (Wiebers et al., 2003). ISUIA data has its strength and weaknesses. However, general consensus among neuro-interventionalists is that most aneurysms $>3 \mathrm{~mm}$ should be treated if it can be done safely. Second, treatment of asymptomatic carotid artery stenosis is shown to be beneficial in ACAS trial (ACAS Trial investigators, 1995). Although no such trial for stenting versus medical therapy in asymptomatic carotid atherosclerosis exists. In some cases, the reason for CAS is not necessarily to treat the lesion for stroke risk reduction, but for surgical planning and access to the distal aneurysm. Third, an aneurysm in the anterior circulation with severe ipsilateral carotid stenosis may be partially protected due to reduce blood flow and in turn reduced pressure head. Recanalization of the carotid artery may increase the flow and in theory may increase the rupture risk. Therefore, once the decision has been made to treat a carotid artery stenosis, any distal aneurysms in its territory should also be secured.

Possible complications with such a treatment include displacement of the stent caused by traversing the guide catheter or other devices across a freshly laid stent. The carotid stent placement commits the patient to immediate and long-term use of antiplatelet agents. In a setting of a ruptured aneurysm, this may add additional risk. Extra radiation exposure is also an additional point to consider.

This series demonstrates that concomitant or single-session coiling of a cerebral aneurysm and ipsilateral CAS is safe and feasible and may even be mandated in cases where improved endovascular surgical access is required.

\section{CONCLUSION}

In our case series of 10 patients, there were no immediate complications. All but one patient have had clinical and imaging follow up, showing no new symptoms and no re-stenosis, aneurysm enlargement, or coil compaction. We conclude that this approach is safe and reduces the inherent risks of performing the procedures separately. Treating the stenosis first avoids the repeated insertion and removal of microwires and microcatheters through a stenosed segment.

Concurrent aneurysm rupture and thrombosis of high grade internal carotid artery stenosis: Report of two cases. Surg. Neurol. 47, 532-539.

Wiebers, D.O., Whisnant,J.P.,Huston, J.III., Meissner, I., Brown, R. D. Jr., Piepgras, D. G., Forbes, G.S., Thielen, K., Nichols, D., O'Fallon, W. M., Peacock, J., Jaeger, L., Kassell, N.F., Kongable-Beckman, G. L., and Torner, J.C. (2003). Unruptured intracranial aneurysms: natural history, clinical outcome, and risks of surgical and endovascular treatment. Lancet 362, 103-110.

Conflict of Interest Statement: The authors declare that the research was conducted in the absence of any commercial or financial relationships that could be construed as a potential conflict of interest.
Received: 21 May 2010; paper pending published: 20 July 2010; accepted: 10 August 2010; published online: 12 October 2010.

Citation: Badruddin A, Teleb MS, Abraham MG, Taqi MA and Zaidat OO (2010) Safety and feasibility of simultaneous ipsilateral proximal carotid artery stenting and cerebral aneurysm coiling. Front. Neur. 1:120. doi:10.3389/fneur.2010.00120

This article was submitted to Frontiers in Endovascular and Interventional Neurology, a specialty of Frontiers in Neurology. Copyright (c) 2010 Badruddin, Teleb, Abraham, Taqi and Zaidat. This is an open-access article subject to an exclusive license agreement between the authors and the Frontiers Research Foundation, which permits unrestricted use, distribution, and reproduction in any medium, provided the original authors and source are credited. 